Session 2793

\title{
Undergraduate Research Using the Finite Difference Time Domain Technique for Electromagnetics
}

\author{
José G. Colom Ustáriz, Rafael Rodríguez Solís \\ University of Puerto Rico at Mayaguez
}

\section{INTRODUCTION}

The Department of Electrical and Computer Engineering (ECE) at the University of Puerto Rico at Mayaguez offers five-year B. S. degrees in electrical and computer engineering. In addition, M.S. and M.E. degrees are offered in both programs. The Electrical Engineering program has a strong core from which the students receive a broad spectrum of the fundamental electrical engineering principles. The undergraduate curriculum requires the students to specialize in one of five options: Communications, Controls, Electronics, Power Systems, and the recently formed Applied Electromagnetics option. The Applied Electromagnetics area at the University of Puerto Rico Mayaguez includes undergraduate courses on antenna theory and design, microwave engineering, radio wave propagation, telecommunication electronics, and a capstone design experience. At the graduate level, courses on microwave remote sensing, advanced microwave circuit design and antenna design are offered. Due to the challenging nature of electromagnetic theory, it can be difficult to attract students to this particular area. In the authors' experience, the most successful instrument to attract students is by providing them with undergraduate research opportunities. The Industrial Affiliates Program (IAP) of the ECE Department is one of the programs providing such opportunities. IAP is supported by 13 companies and it has been running continuously for the last 11 years, supporting over 300 undergraduate students. The students participating in the program usually register in Undergraduate Research (INEL 4998), which is a flexible course that can count for up to 3 credit hours a semester for a maximum of 6 credit hours during the student's career. The size of the course is four students in average. The main advantage over a capstone design course is that the student has two semesters to complete the project under close supervision of the advisor. The students are also exposed to state of the art equipment that is usually reserved for research activities. The authors have used this program to offer research experiences in the electromagnetic areas to 22 students in nine different projects for the last three years. In seven of the nine projects the students used the Finite Difference Time Domain (FDTD) technique for electromagnetics to perform different microstrip structure simulations. The concepts behind this technique are simple enough for junior electrical engineering students to grasp. Remcom Inc ${ }^{1}$ has donated the XFDTD program, which has a graphical user interface for the visualization of the structures under study and the electromagnetic fields associated with it. In this paper, a two-semester undergraduate research course sequence using FDTD as the main tool for electromagnetic simulation is presented. 


\section{FDTD BASIC PRINCIPLE}

Microstrip structure problems are complicated to analyze in part due to the presence of the dielectric substrate. The structure is not symmetric since the metallization is placed between the dielectric and free space, therefore there is no pure TEM propagation mode and there is also possibility of radiation. One way to completely analyze the structure is using a three dimensional numerical simulation, where all the electric and magnetic fields can be calculated throughout a volume containing the microstrip circuit while observing their development in time. One of the most attractive methods that provides this type of simulation, and the main CAD tool used in this work, is the Finite Difference Time Domain or FDTD.

This approach was first originated by $\mathrm{Yee}^{2}$ for the simulation of two dimensional electromagnetic scattering problems. His paper also presented a straight forward derivation for the more general three dimensional problem. The FDTD technique has been widely used in the past few years, and several publications provide an extensive analysis of the subject ${ }^{3}$. The FDTD method is directly derived from discretizing the differential form of Maxwell's two curl equations. This direct application makes the code more efficient and at the same time easier to implement. Throughout the years, this method has been used to solve all kind of electromagnetic problems, and in recent years has been successfully applied to simple microstrip geometries ${ }^{4,5,6}$. One of the advantages of this method is that by exciting a microstrip structure with a single pulse, a large amount of information can be obtained from the resulting time domain fields. In this work, the interest is to use the frequency domain S-parameters to completely characterize the microstrip structure. The S-parameters can be easily computed by properly applying the Fast Fourier Transform (FFT) to the collected time domain data. In addition to the S-parameters, the students can easily observe with XFDTD the time variation of the electric and magnetic fields associated to the structure.

The discretization of Maxwell's two curl equations is the basis for the FDTD implementation. These two equations govern the propagation of the electromagnetic wave and they are:

$$
\begin{aligned}
& \mu \frac{\partial \bar{H}}{\partial t}=-\nabla \times \bar{E} \\
& \varepsilon \frac{\partial \bar{E}}{\partial t}=\nabla \times \bar{H}-\sigma \bar{E}
\end{aligned}
$$

The equations can be written in Cartesian coordinates components, where a discrete approximation to the partial differential equations using centered difference approximation is applied to the time and spatial derivatives as,

$$
\begin{aligned}
\frac{\partial f}{\partial x} & \rightarrow \frac{f(x+\Delta x / 2, t)-f(x-\Delta x / 2, t)}{\Delta x} \\
\frac{\partial f}{\partial t} & \rightarrow \frac{f(x, t+\Delta t / 2)-f(x, t-\Delta t / 2)}{\Delta t}
\end{aligned}
$$

To implement the centered difference approximation, Yee defined the position of each of the six discretized field components in the FDTD rectangular unit cell or the Yee cell. This cell has dimensions $\Delta x * \Delta y * \Delta z$ and the electric and magnetic fields locations are interleaved by half of

the discretization length $(\Delta x / 2, \Delta y / 2$, and $\Delta z / 2)$. This arrangement of fields is necessary to obtain 
the centered difference approximations to the spatial derivatives. In a similar manner, by alternatively calculating the electric and magnetic fields every half a time step $(\Delta t / 2)$, the centered difference for the time derivative is achieved. Since the space derivative is evaluated with the half time step in between the two half time steps used to compute the time derivative, this algorithm is known as the "leap frog" algorithm.

An FDTD space is created by stacking several unit or Yee cells in a fictitious rectangular volume. The desired microstrip geometry is constructed inside this volume. The microstrip circuit is reproduced inside the FDTD space following the physical geometry of the structure by assigning to the unit cells the corresponding type of material from which each section of the structure is made.

The FDTD method is straightforward and can be easily coded to use in a computer program. The discretized fields are updated each time step in the FDTD volume without the need of any complex numerical procedures.

\section{COURSE DESCRIPTION}

The undergraduate research course using FDTD is a two-semester course. The first semester is focused in the introduction of basic principles in the areas of microwave/antenna engineering, FDTD, and metamodeling. During this semester the students is exposed to the XFDTD software and start the analysis of the structure under study. During the second semester the students will dedicate most of their time to run simulations and complete their research. In addition, the student will attend to at least two seminars in how to prepare effective oral and poster presentations and written reports.

The core of the course is the application of FDTD to microstrip structures. What follows are the simulation rules discussed in the course. The objective of the course is to parameterize new structures using statistical techniques. The statistical models are developed with the FDTD data and they are known as metamodels. The metamodel is used to quickly predict the frequency response of the structure without the need of running the 3-D electromagnetic simulator.

Figure 1 shows a single microstrip line inside an FDTD computational domain with total dimensions $I \Delta x * J \Delta y * K \Delta z$ (where $I, J$, and $K$ are integer numbers that define the FDTD space size). The XFDTD program was used to create similar and more complicated geometries inside the FDTD space with the help of a graphical user interface. As shown in the figure, the dielectric material is set to take the lower portion of the cube, usually 10 to 20 cells when high resolution is desired. The microstrip line metallization is simulated by assigning a PEC material to the corresponding electric fields on the dielectric/air interface. To simulate the ground plane, the lowest plane of mesh edges (plane $k=1$ ) along the microstrip conductor plane is also set to a perfect conductor. The other five walls from the cube are simulated as an unbounded space by applying an absorbing outer boundary condition (ABC). Enough space between the microstrip conductor line and the FDTD outer walls is necessary for the proper simulation of the unbounded space. The electric field mesh locations on the surface of the substrate also receives a special treatment if they are not set to PEC. To accurately model the air/dielectric interface in the FDTD equations, these fields are assigned a dielectric constant of $\left(\varepsilon_{\mathrm{r}}+1\right) / 2$. It has been also observed 
that due to the approximation in the spatial derivative, the widths of the microstrip line simulated with FDTD are actually extended by approximately $1 / 2$ a cell at each side of the line. This approximation has to be taken into account when constructing microstrip circuits with FDTD, especially when narrow line widths and spacings are present in the structure. By reducing the cell size, the spatial resolution increases and the error becomes less critical. This approximation also applies to the length of the line, yet is not as critical since the error is negligible when compared to a long line. Special care must be taken when constructing the lines to compensate for this error. For example, to simulate a $1 \mathrm{~cm}$ width line with $\Delta=0.2 \mathrm{~cm}$, four cells are required instead of five. The actual FDTD width of the line can be calculated as $4 * \Delta+2(1 / 2 * \Delta)$. Finally, since $98 \%$ of the fields are contained within two widths of the microstrip line, a distance of $2 * W$ is used in FDTD to separate the microstrip line and the FDTD walls (see Figure 1). Usually, the space left for the proper operation of the ABC will take care of this condition.

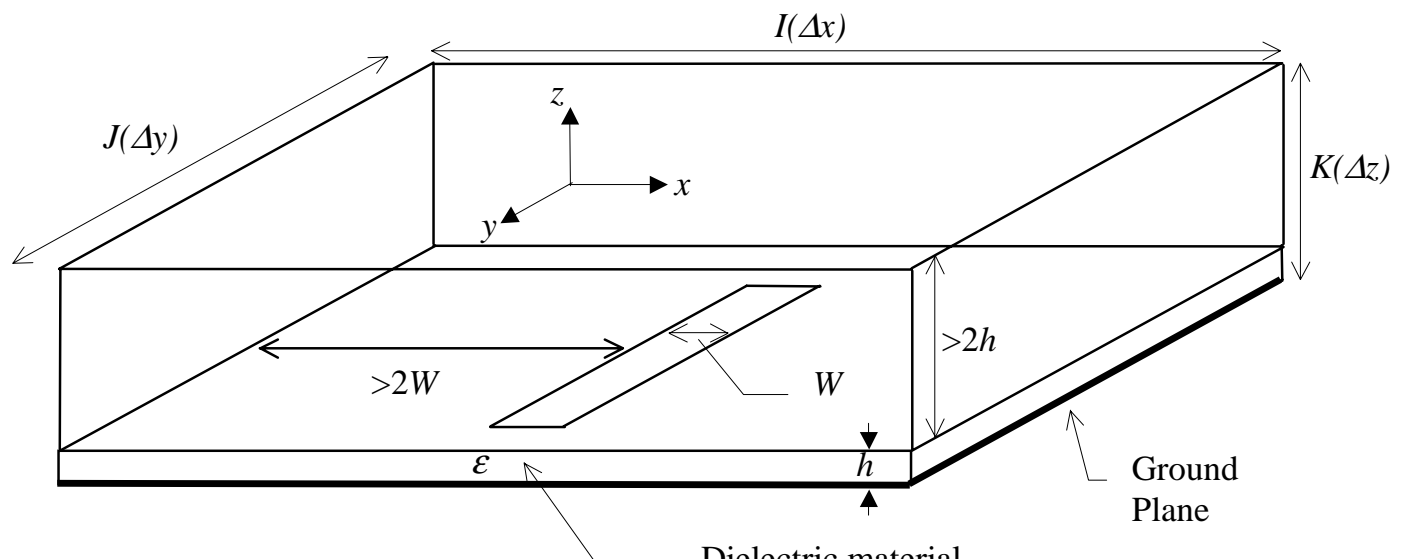

Dielectric material

FIGURE 1 - FDTD space for a single microstrip line.

In XFDTD, the source location is selected by specifying the coordinates $\left(i_{\mathrm{si}} \Delta \mathrm{x}, j_{\mathrm{si}} \Delta \mathrm{y}, k_{\mathrm{si}} \Delta \mathrm{z}\right)$ within the FDTD space. This source location is usually at the ends of the microstrip lines, this is shown in Figure 2 for a single microstrip line segment. The arrow in the figure represents the location of the source and the direction of the field applied.

In most cases, a Gaussian pulse in the $+\mathrm{z}$ direction is used to excite the microstrip circuit. However, if energy is trapped in the structure at undesired frequencies (this has been observed for spiral antennas and for the Lange coupler) excessive time steps can be required for the total energy release. To avoid this problem and speed up the FDTD run a modulated Gaussian pulse should be used. The frequency bandwidth and center frequency for this pulse can be controlled so that the microstrip structure is excited only at the desired frequencies. 


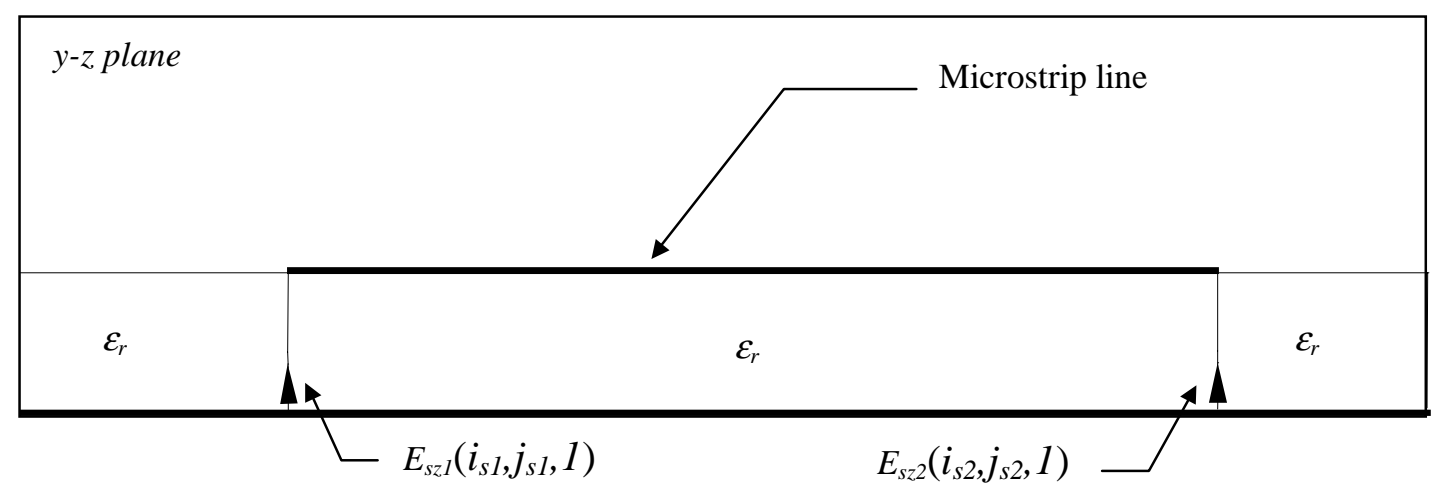

FIGURE 2 - Source locations in microstrip line.

Once the students understand the basic simulation guidelines, they are able to simulate the structures. The students will observe the frequency response of the structure by computing the $\mathrm{S}$-parameters from the time domain simulation data. The dimensions are methodically varied to parameterize the output response using the Design of Experiments (DoE) technique.

A typical Undergraduate Research course using FDTD covers the following topics:

- Basic concepts in antenna/microwave circuits

- Microstrip

- S-parameters

- Maxwell Equations in the time domain

- Discretization of Maxwell Equations

- Finite Difference Time Domain Technique

1. Yee Cell

2. Courant Stability Criteria

3. Boundary Conditions (Liao, PML)

4. Implementation of Microstrip Structures using FDTD

5. Source Excitation

6. Fast Fourier Transform

7. Computation of S-parameters

- Statistical Techniques

1. Design of Experiments (DoE)

2. Metamodeling

The goal of the course is for the students to perform research at an undergraduate level. At the end of the course the student will be able to define a work-plan, timely follow a work-plan, clearly communicate ideas both in written report and oral presentations, facilitate effective interpersonal relationship within a team, effectively used CAD tools (XFDTD) for the simulation of passive structures, clearly and effectively design and conduct experiments, interpret the gather data and report the results, and effectively search technical journals and books for references for their research. In addition the students will be expose to state of the art equipment to perform their research and will have a better idea of what to expect at graduate school. 
To assess the student learning outcomes one final report will be requested at the end of each semester, the student will meet with their advisor for weekly oral progress reports and they will present their work in two local conferences during the second semester.

\section{PROJECT EXAMPLE}

In this section a microstrip antenna project is presented. The results of the research were presented at the IEEE International Antenna and Propagation Symposium in June 2000. The objectives of this research are to alleviate the problem of the propagation of surface wave modes in microstrip patch antennas by routing a trench around the periphery of the patch and to
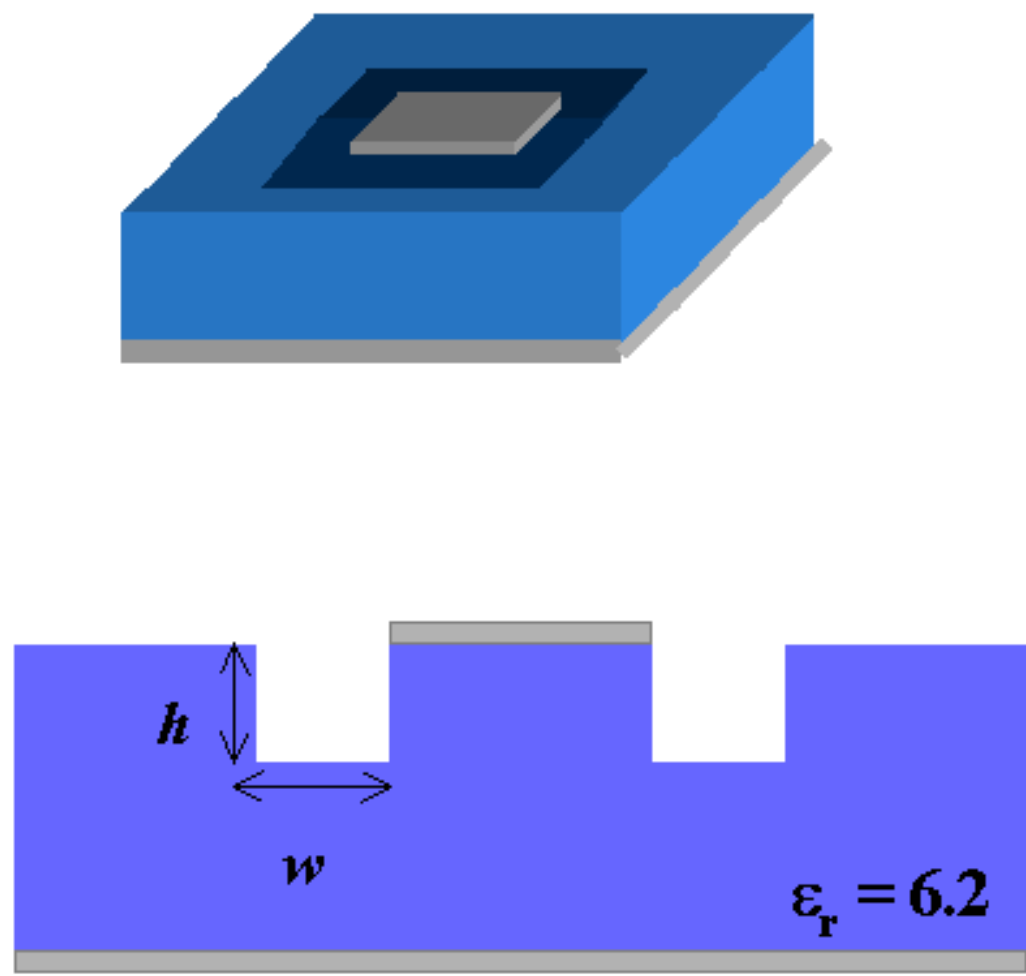

FIGURE 3 - Geometry of microstrip patch

study the effects caused by the trench in the patch input impedance. To achieve the objectives a trench or channel of width $w$ and depth $h$ is cut in the substrate around the periphery of the microstrip patch as shown in Figure 3.

The antenna was designed for an operating frequency of $12 \mathrm{GHz}$ on a substrate with relative permittivity of 6.2 . The substrate thickness was $2.54 \mathrm{~mm}$, giving a patch size of $3.15 \mathrm{~mm} \times 3.15$ $\mathrm{mm}$. 
A 3-level, 2-variable full-factorial Design of Experiments was used. The variables selected were the width and the depth of the cut $(w, h)$. The 3 levels for the width were selected as $0.3 \mathrm{~mm}, 0.6$ $\mathrm{mm}$, and $0.9 \mathrm{~mm}$, while the levels for the length variation are $0.635 \mathrm{~mm}, 1.27 \mathrm{~mm}$, and $1.91 \mathrm{~mm}$. A total of nine simulation runs resulted for this particular DoE. All runs were performed in XFDTD.

The FDTD model for this structure has the following parameters:

- $\Delta \mathrm{x}=\Delta \mathrm{y}=0.16 \mathrm{~mm}, \Delta \mathrm{z}=0.2117 \mathrm{~mm}$

- Gaussian derivative pulse, $500 \Delta t$ long at the Courant stability condition

- Patch size: 20x20 cells

- Substrate: 25 cells around the patch, 12 cells thick

- 10 cells free space border, 8 layer PML ABC

After the simulation is completed, time variations of the electric and magnetic fields can be observed. Figure 4 shows the magnitude of the electric fields for a patch without the trench and Figure 5 shows the magnitude of the electric field with a trench cut in the substrate. Note that the electric field intensity is higher underneath the patch for the patch with the trench. This means that there is less energy getting coupled into surface wave modes for this type of structure.

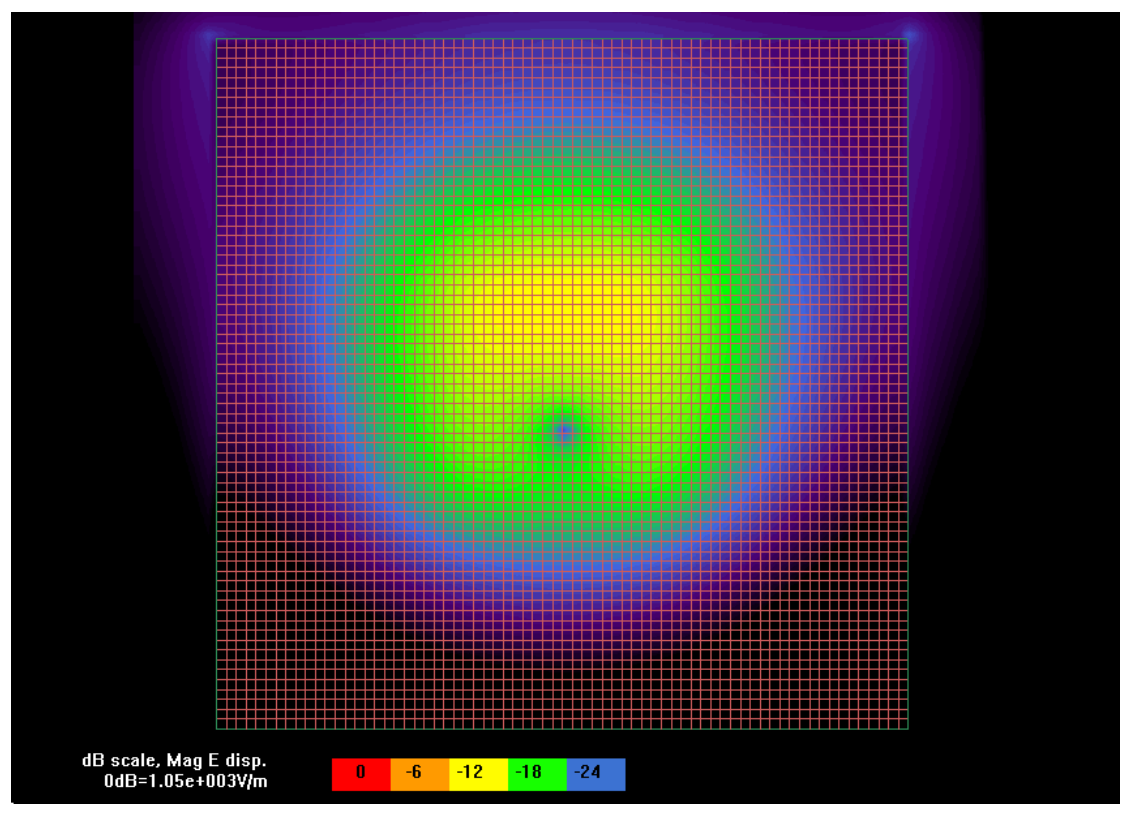

FIGURE 4 - Magnitude of electric fields for a patch antenna. 


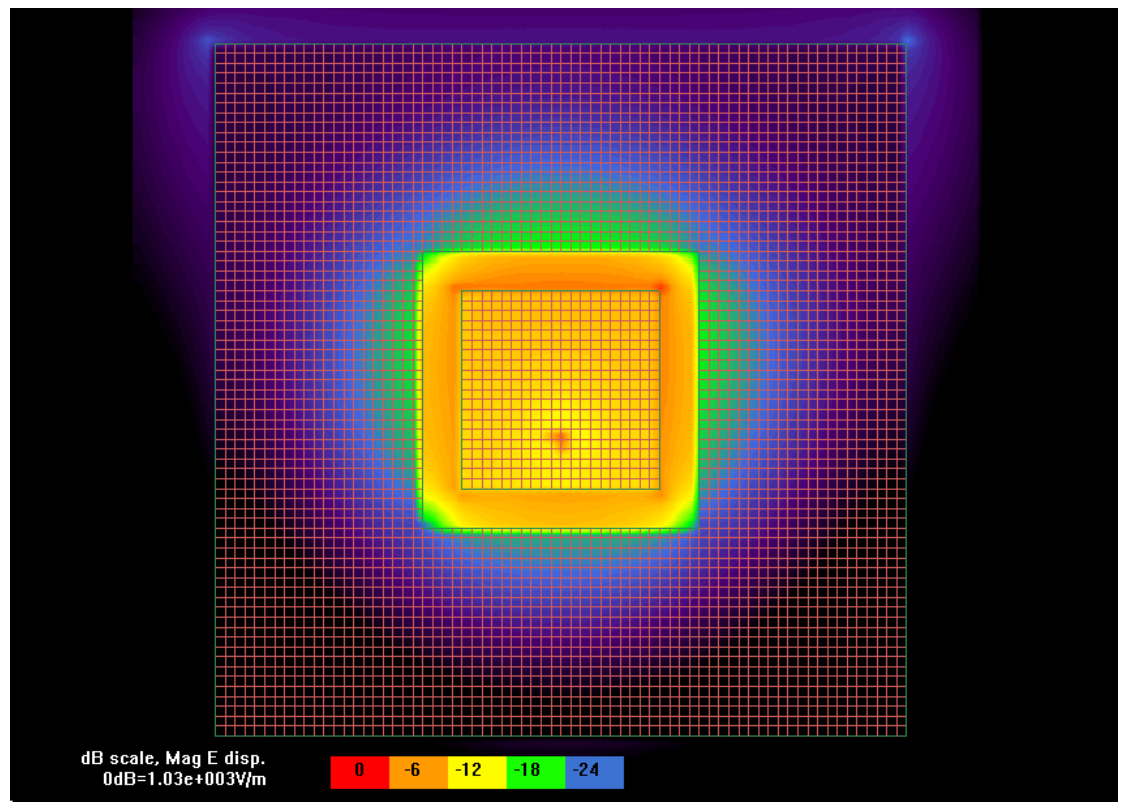

FIGURE 5 - Magnitude of electric fields for a patch antenna with the trench.

S-parameters were also computed $\left(\mathrm{S}_{11}\right)$ for the nine DoE runs, and a polynomial metamodel was developed to predict the output frequency response of the structure.

\section{CONCLUSIONS}

Students registered in the two-semester Undergraduate Research course have the opportunity to study a passive microstrip structure using a 3-D electromagnetic simulator. This undergraduate research opportunity provides them with the experience of applying junior level electromagnetic theory to practical problems. The direct implementation of Maxwell equations using the FDTD method is easily visualized by the students with XFDTD, and it is a strong indication of how important and powerful the electromagnetic theory can be. This experience, usually increases their interest to follow a specialization in the Applied Electromagnetics area at UPRM.

\section{BIBLIOGRAPHY}

1. REMCOM, Inc., User's Manual for XFDTD, Mar. 1997

2. Yee, K.S., "Numerical Solution of Initial Boundary Value Problems involving Maxwell's Equations in Isotropic Media," IEEE Trans. Antennas Propagat., Vol. AP-14, pp. 302-307, May 1966.

3. Kunz, K.S. \& R.J. Luebbers, Finite Difference Time Domain Method for Electromagnetics, CRC Press, FL, 1993.

4. Sheen, D.M., S.M. Ali, M.D. Abouzahra, \& J.A. Kong, "Application of the three-dimensional finite difference time-domain method to the analysis of planar microstrip circuits," IEEE Trans. Microwave Theory \& Tech., Vol. MTT-38, pp. 849-857, Jul. 1990.

5. Shibata, T. \& H. Kimura, "Computer-aided engineering for microwave and millimeter wave circuits using FDTD technique of field simulations," Int. J. Microwave Millimeter-Wave Computer-Aided Eng., Vol. 3, pp. 238-250, 1993.

Annual Conference \& Exposition Copyright $\odot 2001$, American Society for Engineering Education Proceedings of the 2001 American Society for Engineering Education 
6. Lin, X.P. \& K. Naishadham, "A Simple Technique for Minimization of ABC-Induced Error in the FDTD Analysis of Microstrip Discontinuities," Betz, IEEE Microwave \& Guided Wave Letters, Vol. 4, pp. 402-404, Dec. 1994.

\section{JOSE G. COLOM USTARIZ}

Jose is currently an Assistant Professor at the University of Puerto Rico Mayaguez. He has a Ph.D. in Electrical Engineering from Penn State University and an MSEE from UMASS at Amherst. His research interest are microwave circuits, microwave remote sensing, and numerical methods for electromagnetics. He is currently the coordinator of the COOP program at the ECE Department and the IAP coordinator. He teaches courses in the area of Applied Electromagnetics and basic circuit analysis.

\section{RAFAEL RODRIGUEZ SOLIS}

He is an Assistant Professor at the University of Puerto Rico in Mayaguez. He has a Ph.D. in Electrical Engineering from Penn State University and an MSEE from the University of Florida. His research interest are microwave broadband antennas, microwave circuits, high frequency simulations, and numerical methods for electromagnetics. $\mathrm{He}$ is currently the director of the Radiation Laboratory, which is sponsored by NSF under the MRI program. 